

\title{
INTERNAL DESIGNS APPLICATION FOR INLET AND NOZZLE AIRPERFORMANCE IMPROVEMENT
}

\author{
M. Gilinsky ${ }^{\dagger}$ \\ Hampton University, Hampton, VA 23668 \\ and \\ I.M. Blankson" \\ NASA Glenn Research Center \\ Cleveland, $\mathrm{OH} 44135$
}

\begin{abstract}
The following research results are based on development of an approach previously proposed by the authors for optimum nozzle design to obtain maximum thrust. The design was denoted a Telescope nozzle. A Telescope nozzle contains one or several internal designs of certain location, which are inserted at certain locations into a divergent conical or planar main nozzle near its exit. Such a design provides additional thrust augmentation over $20 \%$ by comparison with the optimum single nozzle of equivalent lateral area. What is more, recent experimental acoustic tests have discovered an essential noise reduction due to Telescope nozzles application. In this paper, some additional theoretical results are presented for Telescope nozzles and a similar approach is applied for aeroperformance improvment of a supersonic inlet. In addition, a classic gas dynamics problem of a similar supersonic flow into a plate has been analyzed. In some particular cases, new exact analytical solutions are obtained for a flow into a wedge with an oblique shock wave. Numerical simulations were conducted for supersonuc flow into a divergent portion of a $2 \mathrm{D}$ or axisymmetric nozzle with several plane or conuical designs as well as into a $2 \mathrm{D}$ or axisymmetric supersonic inlet with a forebody. The Ist order Kryko-Godunov marching numerical scheme for inviscid supersonic flows was used. Several cases were tested using the NASA CFL3d code based on full Navier-Stokes equations. Numerical simulation results have confirmed essential benefits of Telescope design applications in propulsion systems.
\end{abstract}

\section{INTRODUCTION}

Several well-known experimental results show essential acoustic benefits in the application of some untraditional nozzle designs. For example, nozzles with rectangular or elliptic cross section in the supersonic part produce less jet noise than round nozzles designed for a fixed Mach number at the nozzle exit (i.e. with uniform flow at the exit and pressure coinciding with the flight static pressure outside the exhausting jet). Thus, the theoretical perfectly shock free jets are "noisier" than

† Research Professor, Senior Member AIAA

* Senior Scientist/Technologist, Associate Fellow AIAA at least partially underexpanded (or overexpanded) jets with possible internal shocks. Moreover, the experimental research of Ahuja, Krothapalli et al. $[1,2]$ has shown that inserting disturbing elements into supersonic jet flow: slots, finger, tabs etc., can reduce jet noise (and screech tones) in spite of the presence numerous strong and weak shock waves. This contradicts the traditional view on the considered phenomenon. A reasonable explanation for these facts would be the appearance of more effective mixing and destruction of the regular cell-shock structure in the weakly underexpanded jet. Inside such a jet, the weak barrel-shaped shock waves are always present and these shock waves are the main sources of the oscillatory processes in the jet. In the regular almost parallel co-annular mixing layers, unstable longitudinal waves are excited, and noise is produced in the fixed direction of the jet axis $\sim 145^{\circ}$. Of course, the presence of shock waves in the jet exhaust, especially for a supersonic nozzle, can lead to some dangerous side effects and performance penalties.

Developing previous ideas for jet noise reduction, two novel concepts were proposed in the papers [3-6]. This first concept is denoted as the Telescope nozzle, for it consists of several internal nozzle surfaces that are arranged in a telescope fashion. The second concept is denoted as the Bluebell nozzle, based on the flowerlike shape of its external jet plume. Bluebell nozzles utilize both chevrons and corrugation in its nozzle geometry. Each concept is capable of achieving a thrust performance greater than the standard baseline conic or $2 \mathrm{D}$ plane convergent and convergent-divergent (CD) nozzles. The improved performance for Bluebell nozzles occurs due to increase in nozzle internal surface area while maintaining nozzle-projected area equivalent to the baseline reference nozzle. Small scale and large scale acoustic tests of different modifications of Bluebell nozzles were conducted at the NASA Langley Research Center and Central AeroHydrodynamics Institute (TsAGI) in Moscow, Russia. These tests have shown essential acoustic benefits Bluebell design application in supersonic regimes as well as in subsonic regims. For example, the experimental tests of several Bluebell nozzle designs ([3]) have shown noise reduction 
relative to a CD round nozzle with design exhaust Mach number $M_{e}=1.5$. The best design provides an acoustic benefit near $4 \mathrm{~dB}$ with about $1 \%$ thrust augmentation. Below, we consider only the first (Telescope) concept with the goal of the Telescope nozzle design optimization for the muximum nozzle thrust, with the intent application of this concept to propulsion systems, especially, for a supersonic engine inlet. Detailed information about the second (Bluebell) concept is in the papers [3-6] and in the patent [7].

\section{PLANE ELEMENT IN SUPERSONIC FLOW}

2.1. The thrust on a plate element with an oblique shock wave and Prandtl-Meyer rarefaction flow. A divergent flow can act on a plate or airfoil inserted into a flow so that a resulting force is directed against the flow. This effect is used for thrust by supersonic nozzles. Conversely, a uniform flow produces only drag for bodies and airfoils. Inserting a conical or wedge-shaped nozzle inside the divergent part of an external nozzle so that the integral of the pressure on the low side of the inserted surface is greater than on the upper side produces increased thrust. There is an optimal angle of the plate that provides the maximum thrust at each point of a divergent flow. The most efficient internal design is produced from a pattern that looks like a telescope with extending tubes. The optimal number of internal designs is defined through dependence on the Mach number at the nozzle exit, $M_{e}$. Telescoping designs must be located so that the compressible waves formed by interaction of a flow with this design would be passed on to the upper side of the next lower telescoping part. The best result will be produced by such a set if the external design inclination increases downstream. Computations show that a significant thrust benefit from the Telescope nozzle occurs with an external telescoping design, using either wedge, conical or optimal contour shapes, and also in the case of a plug application.

This effect can be demonstrated by consideration of the classical steady supersonic flow which forms when an infinite uniform supersonic flow diverges at the deviated wall (convex angle wall). The schematic wave picture of such a flow is shown in Figure 1. A supersonic flow with Mach number, $M=M_{\infty} \geq 1$ is flowing along a rectilinear wall EO. After point $O$, the wall is turned at some angle $\delta_{0}$. The flow velocity increases in the centre wave so that all flow parameters depend only on the angle $\varphi$. All flow parameters can be calculated by:

$$
\begin{gathered}
\lambda^{2}=1+\frac{2}{\kappa-1} \sin ^{2}(a \varphi), \quad \frac{p}{p_{o}}=\left(1-a^{2} \lambda^{2}\right)^{b} \\
M=\sqrt{\frac{\frac{2}{\kappa+1} \lambda^{2}}{1-a^{2} \lambda^{2}}}, a=\sqrt{\frac{\kappa-1}{\kappa+1}}, b=\frac{\kappa}{\kappa-1}
\end{gathered}
$$

$$
\begin{gathered}
w_{r}=\frac{1}{a} \sin (a \varphi), \quad w_{\varphi}=\cos (a \varphi) \\
\sin \alpha=\frac{1}{M}, \delta=\alpha+\varphi-\frac{\pi}{2}
\end{gathered}
$$

where

$$
\begin{gathered}
\lambda_{\infty}^{2}=1+\frac{2}{\kappa-1} \sin ^{2}\left(a \varphi_{\infty}\right), \quad \sin \alpha_{\infty}=\frac{1}{M_{\infty}} \\
\varphi_{0}=\varphi_{\infty}+\alpha_{\infty}
\end{gathered}
$$

Here the angle $\varphi$ is measured from the straight line $\varphi=$ 0 which is inclined to the initial wall direction by the angle $\varphi_{0} ; \delta$ is the angle between local velocity and initial wall direction.

Let a plate element be inserted into this PrandtlMeyer rarefaction wave. Then depending on this element's location and inclination to the local velocity vector, $\mathbf{W}\left(w_{r}, w_{\varphi}\right)$, two different cases are possible. The first, when the element is located in the uniform flow region (the region 5 in Figure 1). Then the flow at the plate is similar and the thrust (or drag) produced by this element can be calculated by relationships in the Prandtl-Meyer rarefaction wave (1-6) for one side of the plate and by relationships on the shock wave (BC) for the other side:

$$
\tan \gamma=\frac{f(\beta) / \tan \beta}{1-f(\beta)}, f(\beta)=\frac{2}{\kappa+1}\left(\sin ^{2} \beta-M^{-2}\right)
$$

where $\beta$ and $\gamma$ are local angles between the flow direction (the vector $\mathbf{W}$ ) and the shock wave and plate respectively. This case is analyzed in paper [3]. It was shown that the optimal inclination of the plate depended on the local Mach number and flow direction. But these results were not connected with any fixed flow, so that the two given parameters, Mach number and angle of flow direction are independent. In the case of rarefaction flow, these parameters are dependent by relationships (1-6). Calculations show that maximal thrust is produced by the unit plate element when this plate is inclined to the initial wall direction of the angle, $\gamma \sim 10^{\circ}$.

In the second case the plate is located in the rarefaction wave and the flow at the plate is not similar. The unknown oblique shock wave is not rectilinear, and the flow behind it is not uniform and parallel to the plate direction. In some approximations, this problem has an analytical solution. For example, the flow on both sides of the plate can be calculated in acoustic approximation for weak waves using an analytical or any numerical method for 2D flow.

2.2. Oblique shock relationship analysis. Some theoretical analysis of shock wave relationship (7) was conducted with the goal to simplify and increase the 
efficiency of the numerical algorithms. Let a plate (or wedge) be located at the angle, $\gamma$ to the supersonic flow direction. It is well known that the formula (7) defines this angle implicitly through use of the angle, $\beta$, between the shock wave and downstream flow direction. Usually, the plate or wedge angle, $\gamma$, is given, and the shock wave angle, $\beta$, is unknown, i.e. its value is calculated by iteration of (7) or by interpolation of the tabled curves $\gamma=F\left(\beta, M_{\infty}\right)$ where $F$ is some function. Of course, explicit formulae are preferable, especially, in more complicated algorithms, which calculate this problem solution repeatedly as, for example, in modern numerical schemes with the Riemann solver. The equation (7) can be transformed to a cubic equation of the variable $\mathrm{y}=\mathrm{f}(\beta)$. For simplification, substitute also: $\mathrm{x}=\tan ^{2} \gamma, \mathrm{a}=(\kappa+1) / 2, \mathrm{~m}=M_{\infty}^{-2}$. Then this eqation becomes:

$$
A y^{3}+B y^{2}+C y+D=0
$$

where $A=a(1+x), B=m-1+(m-2 a) x, C=(a-2 m) x, D=m x$

Explicit solution of this equation can be given using Cordano formulae (see [8]). In accordance with the analysis of the coefficients in (8), we conclude that this equation has three real roots. The simpliest representation of these roots can be given by introduction of the auxiliary angle. Only two positive roots are in the range of the variable $y$. The greater root corresponds to a subsonic value of shock wave inclination, and lower is for a supersonic value. Since these formulae for roots are combersome, we omit them in this paper.

Two important relationships can be found from (7) without use of its exact solution. The first is definition of limit values for the angles $\gamma$ and $\beta$. They are determined at the maximum value of the function $\beta=\beta(\gamma)$, i.e. using the relationship: $d \beta / d \gamma=0$. Equating the derivative of the variable $\beta$ on the right side in (7) to zero, we get the square equation for a variable $z=\sin ^{2} \beta$ :

$$
\kappa z^{2}-(a-2 m) z-\left(m^{2}+a m\right)=0
$$

and positive root is

$$
z=\frac{1}{\kappa}\left(\frac{a}{2}-m+\sqrt{\left(\frac{a}{2}-m\right)^{2}+\kappa\left(m^{2}+a m\right)}\right)
$$

so that limited shock inclination $\beta_{l i m}$ is calculated as $\beta_{l i m}=a \sin (\sqrt{z})$ and the limited wedge inclination $\gamma_{l i m}$ is calculated using formulae ( 7 ) with $\beta_{\text {lim }}$ on the right side. For hypersonic flows with Mach number, $M_{\infty}=$ $\infty(m=0)$ we have:

$$
\gamma_{l i m}=a \sin (1 / \kappa), \quad \beta_{l i m}=a \sin \sqrt{\frac{\kappa+1}{2 \kappa}}
$$

The corresponding curves for dependance of limit angles $\gamma$ and $\beta$ on Mach number and specific heat ratio $\kappa$ are shown in Figures 2 which were calculated using $(10)$ and (7).

The second relationship is for hypersonic flows with Mach number, $M_{\infty}=\infty$. In this case, the solution comes to a solution of the square equation relative to variable, $z=\sin ^{2} \beta$. Designate $t=\tan \gamma$, then the roots of this equation are:

$$
z_{1,2}=\frac{1+(\kappa+1) t \pm \sqrt{1-\left(\kappa^{2}-1\right) t^{2}}}{2\left(1+t^{2}\right)}
$$

where "plus" sign corresponds to subsonic conditions and "minus" to supersonic condition. The set of well known "apple-like" curves for dependence angles $\beta=F(\gamma)$ for different specific heat ratios are shown in Figure 3 . The black poins with numbers 1-11 correspond to limited values of these angles.

2.3. Optimum plate location. In common case, a plate (or airfoil) inserted into an inviscid supersonic flow produces a resulting force normal to the plate and its value and direction depend on the pressure difference on both sides of the plate. The nondimensional aerodynamic characteristics of the plate, the thrust, $T_{n}$, or drag, $C_{D}$, produced by this flow about the plate can be calculated with these four parameters: specific heat ratio $\kappa$, flow Mach number, $M_{\infty}$, an angle $\alpha$ between the flow and thrust direction, and the angle $\gamma$ between the flow and the plate. Schematic geometry and designations for such a flow are shown in Figure 4a. An angle, $\gamma$, is measured from the upstream flow direction. If $\gamma \geq 0$ and less than the limited angle $\gamma_{l i m},\left(0 \leq \gamma \leq \gamma_{l i m}\right)$, the thrust (drag) is determined by the simple analytical formulae using relationships for oblique shock waves and the Prandtl-Meyer rarefaction wave discussed in the previous sections. In this interval of the angle $\gamma$, for all another parameters there is an optimal value of the angle $\gamma_{\text {opt }}$, which gives the thrust maximum value. Aerodynamic characteristics of the unit plane element in supersonic flow were calculated using the created code for a wide range of the parameters: $\mathrm{k}, M_{\infty}$, and $\alpha$. Two examples are illustrated in Figures $4 b, c$ by the curve families for the nondimensional variables $\left(T_{n}, \gamma^{\circ}\right)$ for Mach number $M_{\infty}=2$ and $M_{\infty}=6$ respectively. Numbers 1,2,3,4, and 5 at the curves correspond the angls, $\alpha=0^{\circ}, 22.5^{\circ}, 45^{\circ}, 67.5^{\circ}$, and $90^{\circ}$. Note, that for large attack angles to the thrust direction, $\alpha$, maximum thrust is obtained at the limited angles, $\gamma_{l i m}$.

Similar results were observed for another case that correspons to a pure Prandtl-Meyer flow at the turning point of the 2D nozzle wall. The schematic picture of such flow and designations are shown in Figure 5a. Tipical examples for Mach number, $M_{\infty}=1.5$ and 
3 are illustrated in Figure $5 b, c$. Here, the nondimensional thrust $T_{n}$ vs wall inclination angle, $\gamma$, for several slopes of the flow (or upstream wall) is given. Numbers $1,2,3,4$, and 5 on the curves correspond to angle $\alpha=0^{\circ}, 10^{\circ}, 20^{\circ}, 30^{\circ}$, and $40^{\circ}$. Again, for small angles, $\alpha$, there are maximum thrust values inside the interval $0 \leq \gamma \leq \gamma_{l i m}$, and for greater $\alpha$, maximum thrust occurs at the limited value $\gamma_{l i m}$. Similar results were observed with other Mach numbers.

\section{TELESCOPE NOZZLE NUMERICAL SIMULATION RESULTS}

The main numerical simulation results for Telescope nozzle based on the 1st order Krayko-Godunov marching scheme [9] for inviscid supersonic flows were discribed in the papers $[3,5]$. Some of them will be illustrated below. In particular, it was shown that by the standard deformation of single axisymmetric or $2 \mathrm{D}$ nozzle it is possible to obtain only an insignificant thrust augmentation, even using optimal nozzle shape, i.e. $1-4 \%$ by comparison with usual conical (wedge-shaped) nozzle. A Telescope nozzle can increase thrust significantly more.

3.1 Telescope nozzle geometry. Example of two possible Telescope nozzle embodiments are shown in Figure $7 \mathrm{a}$ and $7 \mathrm{~b}$. In Figure $7 \mathrm{a}$, the external nozzle is constructed by giving the fixed contour $z=2(x)$ in the $\mathrm{zx}$-plane and a cross section contour is described by the super-elliptical equation:

$$
\begin{gathered}
(y / a(x))^{n(x)}+(z / b(x))^{n(x)}=1, z=f(x) \\
n(x)=2+H\left(x-x_{*}\right) \cdot n_{e}\left(x-x_{*}\right) /\left(\left(x_{e}-x_{*}\right)\right. \\
c(x)=a(x) / b(x)=1+H\left(x-x_{*}\right) \cdot c_{e}\left(x-x_{*}\right) /\left(\left(x_{e}-x_{*}\right)\right.
\end{gathered}
$$

where the Heaviside function $\mathrm{H}\left(\mathrm{x}-\boldsymbol{x}_{*}\right)$ is defined: $\mathrm{H}(\mathrm{x}-$ $\left.x_{*}\right)=0$ if $x_{0} \leq x \leq x_{*}$, and $\mathrm{H}\left(\mathrm{x}-x_{*}\right)=1$ if $x_{*} \leq x \leq x_{e}$. The subscripted indicies $0, *$ and e correspond respectively to the nozzle inlet, throat and exit. The subsonic portion of the nozzle (from the inlet to the throat) has axisymmetric shape $(a=b=1, n=2)$. In the supersonic portion (from the throat to the nozzle exit), a power $n$ in (12) changes from the minimal throat value of 2 to the maximal exit value $n_{e}$ and an eccentricity $c=a / b$ changes from the minimal throat value of 1 to the maximal exit value $c_{e}$. The nozzle contour $\mathrm{z}=\mathrm{f}(\mathrm{x})$, in the plane of symmetry, $y=0$, is a cubic parabola in the subsonic part and then becomes rectilinear with the angle $\alpha=10^{\circ}$. For the Telescope nozzle in Figure $6 \mathrm{a}$, the power $n(x)$ increases from 2 to 10 downstream from the throat to the exit and two plane internal designs located symmetrically supported by the holders into the external design. In Figure $6 \mathrm{~b}$ another style of the Telescope nozzle is shown. The external nozzle is described by a super-elliptical equation with constant values $n=2, a=b=1$, and the internal design is described by the same equation with $a=1, b=0.5$, i.e. cross section has an elliptical shape; the internal design is again supported by holders to the external design. In the both cases, the nozzle contour in XZ-plane, $\mathrm{z}=\mathrm{f}(\mathrm{x})$, contains rectilinear and round intervals with continuous inclinations in the points of discontinuous curvature.

Another embodiment is illustrated in Figure 6c. The external design is a Chisel nozzle. This nozzle can be constructed on the base of any plain nozzle. For the simplest design in Figure 6c, dependence of radius on the azimuthal angle in the cross section is described by a periodic function $r=r(\varphi)$ with a period $T=2 \pi / n_{c}$ : into the first period $r=r_{+}=$const for $0 \leq \varphi \leq \varphi_{1}$ and $\varphi_{2} \leq \varphi \leq T$, and $r=r_{-}=$const for $\varphi_{1} \leq \varphi \leq \varphi_{2}$, where $\varphi_{1}=0.5(T-\Delta \varphi)$ and $\varphi_{2}=0.5(T+\Delta \varphi)$. We call a corrugated surface part a "cavity" or a "convexity" relatively to the internal normal to the nozzle wall. The cavity depth (or convexity height) defined by the equality $\Delta r=r_{+}-r_{-}$, increases along the nozzle centerline from zero at the throat, $x=x_{*}$, to the maximum value at the exit, $\Delta^{r}=\Delta_{e}^{r} \xi$, where $\xi=\left(x_{-} x_{*}\right) /\left(x_{e}-x_{*}\right)$, i.e. this coincides with definition of a corrugation amplitude coefficient $\delta$. The cavity (convexity) width, $\Delta \varphi=$ $\varphi_{2}-\varphi_{1}$, also linearly increases (decreases) downstream from zero (maximum) at the throat to the maximum (zero) at the nozzle exit, i.e. $2 \varphi_{1}=T-\Delta \varphi=\mathrm{T} \xi$. For such a configuration, two expanded flows near the nozzle wall flow into two neighbouring cavities to meet each other at some angle $\alpha$, mutually penetrate and more effectively mix. A flow impulse on the lateral area of the convexities increases the resulting nozzle thrust.

A Chisel nozzle is very convenient to use with a Telescope nozzle as shown in Figure 8 because similar convexities allow the internal design to be maintained. In Figure 6c the main external design (1), a Chisel nozzle, is based on the cone of angle $\alpha=10^{\circ}$ and the internal design (2) has a conical surface.

The Blubell nozzle concept application for jet noise reduction can be also used in the Telescope nozzle concept for external design or for internal design as well as for both. Such an example is shown in Figure $6 \mathrm{~d}$. There a 6-petal internal conical design is installed into an 8-petal external design in which three plane holders maintain its position.

3.2 Numerical simulation results. The numerical simulations were conducted using a modified numerical code based on the lst order explicit numerical marching scheme of Kryko-Godunov [9]. Solutions are obtained using an arbitrary curvilinear coordinate system, and the marching coordinate $\mathrm{x}$ is chosen close to the local streamline. A multi-zone approach and nonuniform grid application were used to obtain results of high resolution in complicated geometric domains. 
One 3D numerical simulation result will be illustrated for a Bluebell shaped Telescope nozzle. In Figure 7a-c, this is the Telescope nozzle with conical external design at an angle $\alpha_{2}=20^{\circ}$ and of internal Bluebell design with frequency of eight corrugations without petals, $\varepsilon=0$ and $\delta_{0}=0.4$. The goal of this numerical simulation is to examine the difference between the thrust of the baseline round conical internal design at an angle $\alpha_{1}=5^{\circ}$ and the Bluebell design thrust. Both designs have the same corresponding cross section areas. The average radius of the Bluebell nozzle is calculated by the baseline radius. Therefore the maximal angle of the Bluebell internal design in the $\mathrm{XOZ}$ plane exceeds $5^{\circ}$, and the minimal in the other plane of symmetry is also less than $5^{\circ}$. Mach contours in the sequential downstream cross sections from the throat to the nozzle exit in Figure 12b,c show intensive development of the swirling flow that is swept into both the internal and external flows. This will favor mixing downstream in the jet exhaust and will reduce jet noise. The thrust augmentation of the Telescope nozzle application in this case is $\sim 10 \%$.

The thrust produced by the conical nozzle divergent portion as a function of the nondimensional length of this portion $\mathbf{L} / r_{*}$ is shown in Figure 8 . In this numerical simulation, the maximal nozzle radius at the exit, $r_{e}$, was fixed at $r_{e}=1.75 r_{*}$ where the subscript * corresponds to the nozzle throat cross section, and $r=r(x)$ is the nozzle contour equation in the meridional plane.

The nondimensional thrust is given $T_{n}=T_{d} /\left(\rho_{*} c_{*}{ }^{2} *\right.$ $\left.r_{*}^{2}\right)$, where $\rho$ is gas density and $\mathrm{c}$ is sound speed. The subscripts are $\mathrm{c}$ for the single conical nozzle without any internal design; "sh"-for the total thrust $\left(T_{s h}=T_{*}+\right.$ $\left.T_{c}\right)$; and " $t$ "-for the Telescope conical nozzle with one internal design which has the length $l_{t}=0.3 \mathrm{~L}$. The internal design is a plate with the entry coordinate $x_{t}^{o}=0.7 \mathrm{~L}$ end coordinate $x_{t}^{c}=x_{e}$, i.e. at the nozzle exit. The entry radius of the plate is calculated from the condition that this entry point is located at the same flow streamline starting at the throat $x_{t}^{*}=0, r_{t}^{*}=0.8$, so that the plate angle is changed as shown in Figure $10\left(\alpha_{t}\right)$. For comparison, the external nozzle angle, $\alpha_{c}$ is also shown in this Figure. It is not at the optimal location and length for maximal thrust. These results illustrate that internal design is effective for short nozzles with relatively large angle $\alpha_{c}$.

The benefit can be more significant for Telescope nozzles with several internal components. Figure 9 illustrates this effect that for three internal components. The thrust benefit here is $\sim 25 \%$. Depending on the optimization conditions, this value can be even higher.

Analogous results were obtained for 2D wedge-shaped Telescope nozzles. Comparison of the thrust for the single nozzle and Telescope nozzle with four internal components has shown that thrust augmentation can approach $\sim 27 \%$. For hypersonic nozzles, this augmentation is again greater. The working efficiency of a Telescope nozzle grows as Mach number increases. For example, the thrust augmentation produced by an internal plate can reach $\sim 75-100 \%$ if this plate would be located in a region of constant parameters of a pure Prandtl- Meyer rarefaction wave. An example of numerical simulation results for the $2 \mathrm{D}$ wedge-shaped nozzle with four internal thin airfoils is shown in Figure 10. Here Mach contours and four streamlines are presented. These streamlines correspond to the internal airfoil stagnation points and also represent the zone boundaries. This picture illustrates the essential benefits of internal design applications inside Prandl-Meyer rarefaction wave and region of the constant parameters at the nozzle wall. In this case, the inlet Mach number is $M_{\infty}=2$, the angle $\beta=30^{\circ}$, and the thrust augmentation for divergent nozzle portion is $h=D T / T \sim 75 \%$. Note that the working efficiency of a Telescope nozzle grows as inlet Mach number increases. For example, for $M_{\infty}=5$, the value of $\mathrm{h}$ can mount to 100 This value can be even larger depending on the angle $\beta$. Experimental measurements of small and large scale Telescope nozzles are being addressed in future work. These experimental tests are very important to conduct because the recent experimental acoustic tests have discovered essential jet noise reduction produced by nozzles with the uniform plate set at the nozzle exit. Such noise benefit was predicted in the papers $[3,5]$.

\section{SUPERSONIC TELESCOPE INLET}

4.1 Supersonic inlet problems. The main purpose of a supersonic inlet is to slow down gas flow and to transfer it from supersonic speed to low subsonic speed before a chamber (compressor). Simultaneously, the total pressure should have minimal loss for effective combustion in a chamber. The first investigations and analysis of this problem took place in the 60's. For $2 \mathrm{D}$ and axisymmetric inlets, the investigations showed that flow total pressure loss through a set of incline oblique shock waves with the last normal shock wave is essentially less than through a unique detached shock wave before the inlet. Several possible inlet flow regimes are shown schematically in Figures $11 \mathrm{a}-\mathrm{h}$ : a) with two shock waves at the inlet plus one external at the cowl; b) three plus one; $c$ ) three with detached shock wave at the cowl; d) continuous compressive waves along a curved inlet surface (forebody) with detached shock wave at the cowl; e) with partial external compression and internal; f) with internal compression; g) with detached shock wave befoe the inlet. Calculations conducted by G.I. Petrov, K. Oswatisch have shown that the total pressure loss in an ideal inlet may be not more than 
$\sim 3-5 \%$ for a shock wave system with equal intensity. In real inlet flow, separation zones can be formed at the sharp change of centerbody inclination or at the point of interaction of the shock wave with the boundary layer at the forebody or cowl. This makes the inlet characteristics worse. Detailed analysis of the supersonic inlet problem is in G.N. Abromovitch's book [10]. Note that most of the optimization theories and numerical simulation methods for improving inlet efficiency do not take these effects into account.

Separation and inlet drag are important obstacles for inlet efficient work. To reduce these effects, application of $3 \mathrm{D}$ corrugated surfaces similar to those that were used for improving nozzle designs may be employed. For example, a star shaped forebody or its smooth modification can reduce forebody drag. Also, it is known from hydrodynamic stability theory (Lin C.C., and others) that $2 \mathrm{D}$ velocity distributions in boundary or mixing layers are less stable than corresponding distributions in $3 \mathrm{D}$ cases because there is one additional degree of freedom for perturbation amelioration. Semi-empirical separation criteria show the same phenomenon. Several unusual curvilinear surfaces were proposed and tested experimentally many years ago by Russian scientists. However, such shapes have not been used in the aviation industry and require further research. Preliminary estimations are very promising.

4.2 Optimum cowl shape analysis. The important inlet problem is to reduce inlet drag, i.e. forebody and cowl. Our calculations, using analytical relationships for shck waves have shown, that inlet drag may be reduced through judicious choice of forebody and cowl location and shapees. A schematic draft of one possible configuration is shown in Figure 14h, where instead of turning oblique shock waves around a cowl front edge, a forebody corner point was used as the turning point. The problem is optimization of the length and angle of cowl front door for a 2D inlet of an air-breathing propulsion system. A systems of three and four oblique shock waves forming at the designed inlet for fixed Mach number with a one and double wedge-shaped forebody were analyzed. The leading edge of a cowl door is the point of crossing of two or three oblique shock waves and the last passes the forebody corner point. The last shock wave joins this corner point and back edge of the cowl door. The shock wave system compresses and turns the initial supersonic flow and again deflects it to the same initial direction. The forebody drag, $C_{D}$, and total pressure losses,$\varepsilon=\left(p_{o}-p_{o e}\right) / p_{o}$, in the cross section behind the forebody corner point were calculated. Here $p_{o}$ and $p_{o e}$ are the total pressures behind normal shock waves upstream of the inlet and behind the corner point. The results are presented as curves for $M_{\infty}$ and $\beta$ (where $\beta$ is an angle between flow and plane cowl door) in the variables $\left(C_{D},\right)$ and $\left(C_{D}, M_{\infty}\right)$ respectively. The main conclusion from this calculation is that for each Mach number there is an optimal value $\beta_{\text {opt }} \geq 0$ which provides a maximum value for working efficiency parameter of such 3 or 4 shocks inlet, $\xi=\left(C_{D} * \varepsilon\right)^{-1}$. The main conclusion for this problem is that the frontal cowl cap can create the additional engine thrust, and wedge shaped forebody drag can be reduced up to $15-20 \%$. Numerical simulations by K-G marching code have shown approximately the same estimations for axisymmetric or 2D inlet with a conical or wedge-shaped forebody nose. The benefits increase with increase a cone (wedge) angle and increase in flight Mach number.

4.3 Some numerical simulation results. The most previous untraditional nozzle designs discussed above can be employed for a supersonic inlet improvement. In particular, a Telescope nozzle and all results of theoretical analysis of this concept are useful. In this case, the energy of the turned flow along the forebody wall can be used for creation of additional thrust as in the previous case with a cowl door. As in the first problem, the mutual locations, sizes and angles of the internal plates (thin airfoils) are very important for efficiency of the application. Optimal values of geometric parameters were determined from multi-parametric numerical simulations based on the modified marching $\mathrm{K}-\mathrm{G}$ code. The effect of four thin airfoils installed at the minimal cross section (nearby of the corner point) is illustrated Figure 12. Here Mach contours and corresponding streamlines are shown for the 2D Telescope inlet with a wedged forebody. This design provides a forebody drag reduction of $25 \%$.

Some previous designs were tested numerically with the purpose of upstream viscous effects influence to the main conclusions. Partially, viscows effects were counted using boundary layer correction in thrust calculation. These estimations were conducted on middle size vehicles for low flight altitudes. Two numerical codes were used: the NASA LaRC CFL3D code [11] and CRAFT-Tech code [12]. Both codes are based on an implicit upwind 2nd order numerical schemes (ENO versions) for solution of the full unsteady and steady Navier-Stokes and Euler equations. An example of a such result is shown in Figure 13. For these conditions (high, Mach and Reynolds numbers), the tests have confirmed the main conclusions of Telescope nozzles and inlets efficiency which were made on the basis of analytical solutions and numerical simulations using the simplified marching scheme Krayko-Godunov [9].

Obviously, the same approach is applicable for other designs, such as transition sections inside variable cross section supersonic tunnels, blunt bodies with several ring-shaped sheets, projectiles, etc. 


\section{CONCLUSION}

Theoretical analysis and numerical simulation results were obtained for nozzles and supersonic inlets with the goal of aeroperformance improvement. The designs investigated are based on development of the approach proposed by the authors for optimum nozzle design for obtaining maximum thrust. Such a design was denoted a Telescope nozzle. A Telescope nozzle contains one or several internal designs at certain locations in the divergent conical or planar main design near its exit. Such design provides additional thrust augmentation over 20$30 \%$ by comparison with the optimum single nozzle of the equivalent lateral area. Recent experimental acoustic tests have discovered essential noise reduction due to Telescope nozzles application as well. Some additional theoretical results were presented for the Telescope nozzle and a similar approach was applied for aeroperformance improvment of a supersonic inlet. At the same time, the classic gas dynamics problem of a similar flow at the plate in a supersonic flow has been analyzed. In some particular cases, new exact analytical solutions were obtained for a flow at the wedge with an oblique shock wave. Numerical simulations were conducted for supersonuc flow into a divergent portion of $2 \mathrm{D}$, axisymmetric and $3 \mathrm{D}$ nozzles with several plane, conuical or corrugated designs as well as into a $2 \mathrm{D}$ or axisymmetric supersonic inlet with a forebody. The 1st order Kryko-Godunov marching numerical scheme for inviscid supersonic flows was used with boundary layer correction in thrust calculation formulae. Several cases were tested using the NASA CFL $3 \mathrm{~d}$ and Tech-CRAFT codes based on the full Navier-Stokes equation. Numerical simulation results have confirmed essential benefits of Telescope design applications in propulsion systems.

\section{ACKNOWLEDGEMENTS}

We would like to acknowledge the NASA Langley Research Center, especially Drs. Dennis M. Bushnell, John S. Preisser, and Joe W. Posey, for interest and support. This research was conducted under the expired NASA grants NAG-1-1835 and 1936, We also are grateful to the NASA Glenn Research Center for the opportunity to continue and develop our concepts under the current NASA grant NAG-3-2249. We would like to thank Dr. John M. Seiner and Dr. Jay C. Hardin for their attention, interest to our research, review and useful suggestions.

\section{REFERENCES}

1. Ahuja K.K., 1993, Mixing Enhancement and Jet Noise Reduction Through Tabs Plus Ejectors, AIAA Paper 93-4347, 15th Aeroacoustics Conference, Oct. 2527, 1993/Long Beach, CA.
2. Krothapalli, A. and King C.J., 1993, The Role of Streamwise Vortices on Sound Generation of a Supersonic Jet, 15th AIAA Aeroacoustics Conference, Oct. 25-27, 1993/Long Beach, CA.

3. Seiner, J.M., and Gilinsky, M.M., 1995, Nozzle Thrust Optimization while Reducing Jet Noise, 26th AIAA Fluid Dynamics Conference, June 19-22, 1995/San Diego, CA.

4. Seiner, J.M., and Gilinsky, M.M., 1995, "Nozzle Thrust Optimization while Reducing Jet Noise", 1st Joint CEAS/AIAA Aeroacoustics Conference, June 1215,1995, Munich, Germany.

5. Gilinsky, M.M., and Seiner, J.M., 1996, Corrugated Nozzles for Acoustic and Thrust Benefits of Aircraft Engines, CEAS/ AIAA Paper 96-1670, 2nd CEAS/AIAA Aeroac. Conf., May 6-8, State College, PA.

6. Seiner, J.M. and Gilinsky, M.M., 1997, Nozzle Thrust Optimization while Reducing Jet Noise, AIAA J, No. 3.

7. Seiner, J.M. and Gilinsky, M.M., 1999, Jet Nozzle Having Centerbody for Enhanced Exit Area Mixing, US Patent $\# 5,924,632$.

8. Brounshtein, I.N., and Semendyaev, K.A., 1981, Mathematical Guide, Nauka, Fizmatgiz, 718p.

9. Godunov, S.K. et al., 1976, Numerical Solution of Multidimensional Problems of Gas Dynamics, Moscow: Nauka, 1976, 400p.

10. Abromovich G.N. Applied Gas Dynamics, 1976, Nauka, Moscow, 888p.

11. Krist, S.L., Biedron, R.T., and Rumsey, C.L., 1996, CFL3D User's Manual (Version 5.0), NASA Langley Research Center, 311p.

12. Molvik, G.A., and Merkle, C.L. 1989, A Set of Strongly Coupled, Upwind Algorithms for Computing Flows in Chemical Nonequilibrium, AIAA Paper 890199, 27th Aerospace Sciences Meeting, Jan. 9-12. 


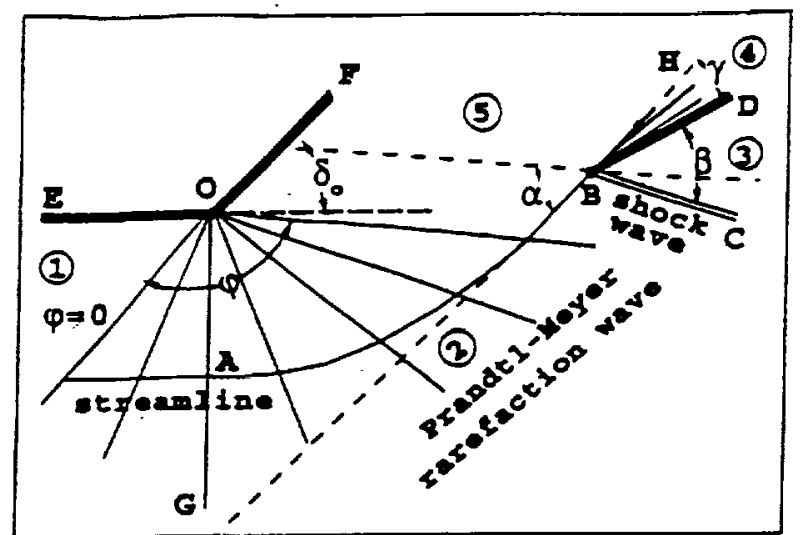

Figure 1. A similar flow in a Prandt1-Meyer rarefaction wave; the thrust of the unit plate element (BD) in this wave. The regions and nomenclature: 1 )supersonic inviscid uniform flow at the rectilinear wall, $2)$ - steady rarefaction wave, $\varphi$-angle of the 1st family characteristic, $\alpha$ - local angle between this characteristic and a streamline; 3 )-uniform flow at the plate behind the oblique shock wave (BC), $\beta$ - angle between the plate element and shock wave; 4)-Prandtl-Meyer rarefaction flow at the plate element, $\gamma$-angle between this element and the streamline; 5)-uniform flow at the wall behind its turn, $\delta_{0}$-turn angle.

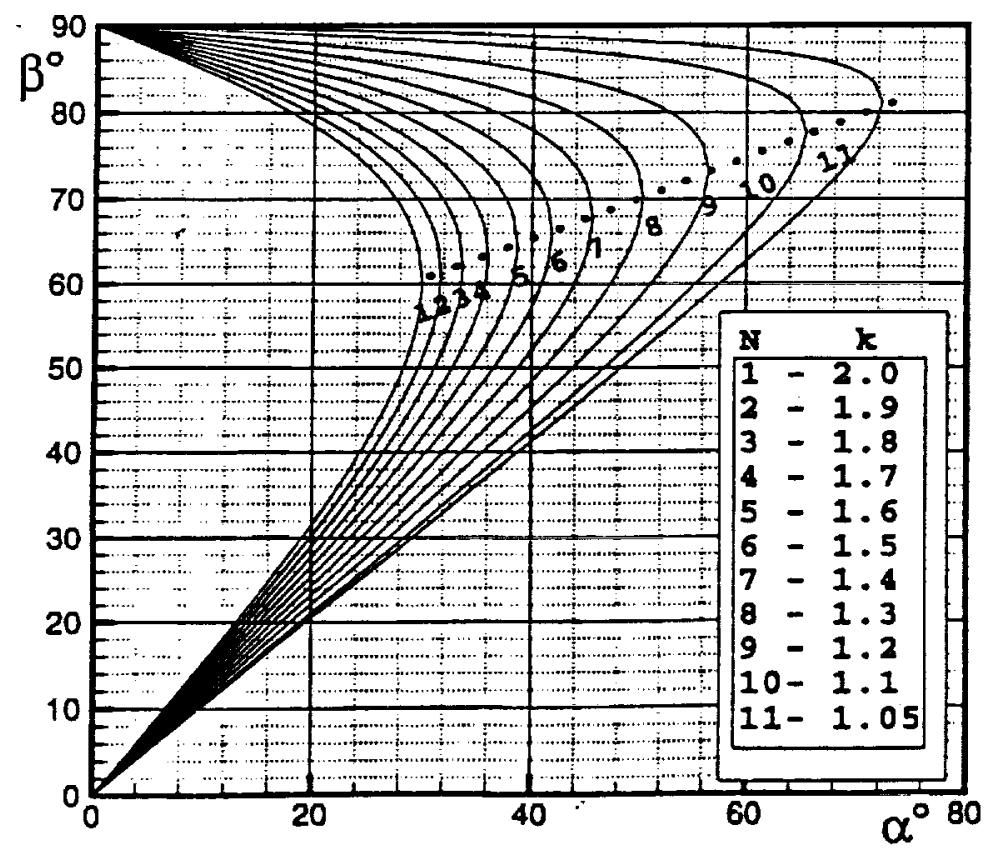

Figure 3. Analytical solution for supersonic flow at the wedge with oblique shock wave for Mach number. $M_{\infty}=\infty$. Shock wave angle, $\beta$ vs wedge angle, $\alpha$.
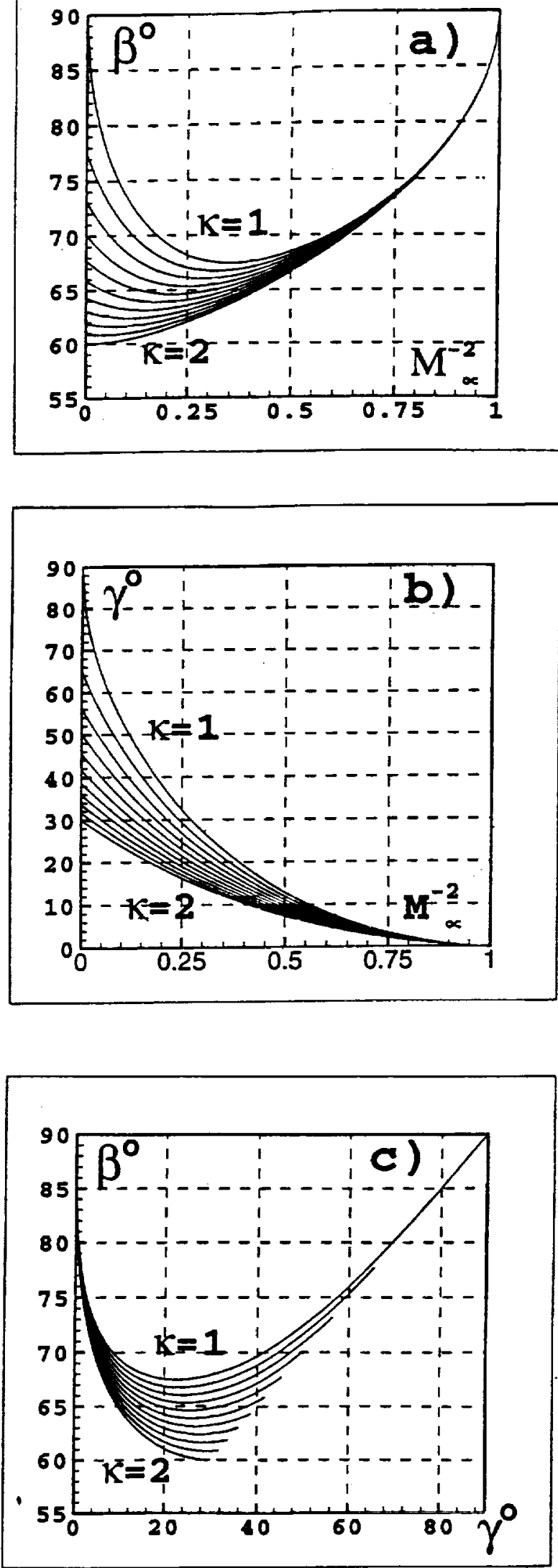

Figure 2. Analytical solution for supersonic flow at the wedge with oblique shock wave: a)-Shock wave angle, $\beta$, vs Mach number function, $M_{\infty}^{-2}$ for different specific heat ratios: $\kappa=1.0,1.1, \ldots, 1.9,2.0 ; \mathrm{b}$-Wedge angle, $\gamma$, vs $\left.M_{\infty}^{-2} ; \mathrm{c}\right)$-Shock wave angle, $\beta$, vs wedge angle, $\gamma$. 

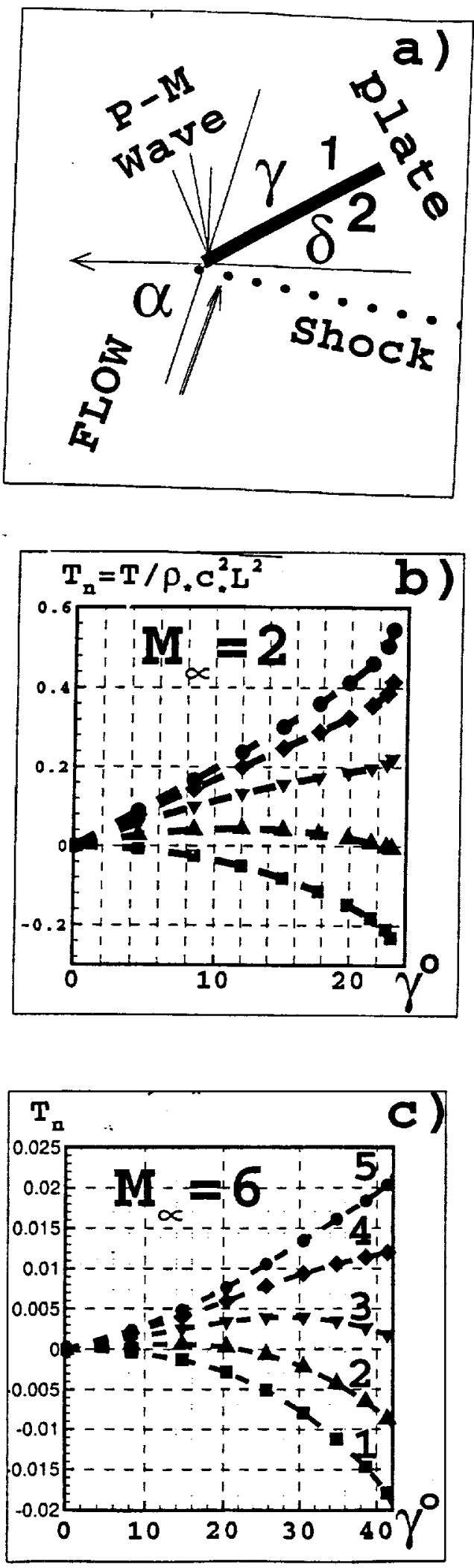

Figure 4. Unit plane element thrust, $T_{n}$, vs flow angle $\alpha$, plate slope, $\gamma$, and flow Mach number, $M_{\infty}$; Analytical solution for supersonic flow at the plate with oblique shock wave and Prandtl-Meyer rarefaction wave: a)- Draft and designations; b) and c)-nondimensional thrust vs plate slope, $\gamma$ for $M_{\infty}=2$ and 6 respectively. Numbers: $1,2,3,4$, and 5 are for flow attack angle $\alpha=0.0$, $22.5,45.0,67.5$, and 90.0 (deg.).
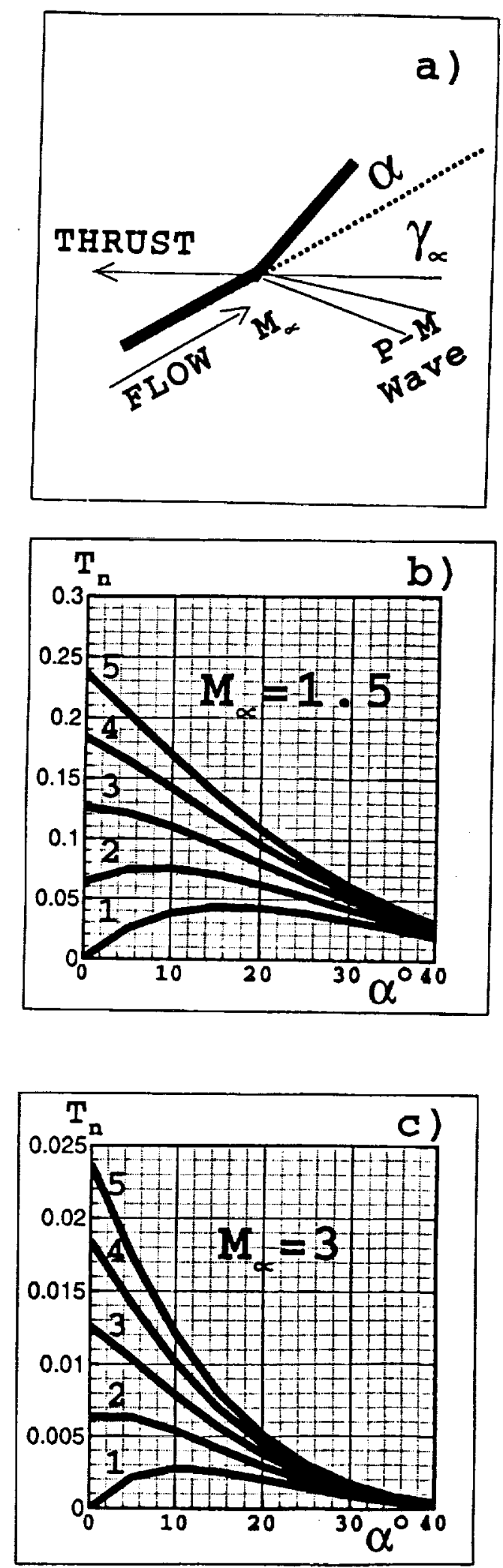

Figure 5. Unit plane element thrust, $T_{n}$, vs flow angle $\gamma_{\infty}$, plate slope, $\alpha$, and flow Mach number, $\dot{M}_{\infty}$; Analytical solution for supersonic flow at the turning wall with the Prandtl-Meyer rarefaction wave: a)-Draft and designations; b) and $\mathbf{c}$ )- nondimensional thrust vs plate slope, $\alpha$ for $M_{\infty}=1.5$ and 3 respectively. Numbers: $1,2,3,4$, and 5 are for flow attack angle $\gamma_{\infty}=0,10,20$, 30 , and 40 (deg.). 

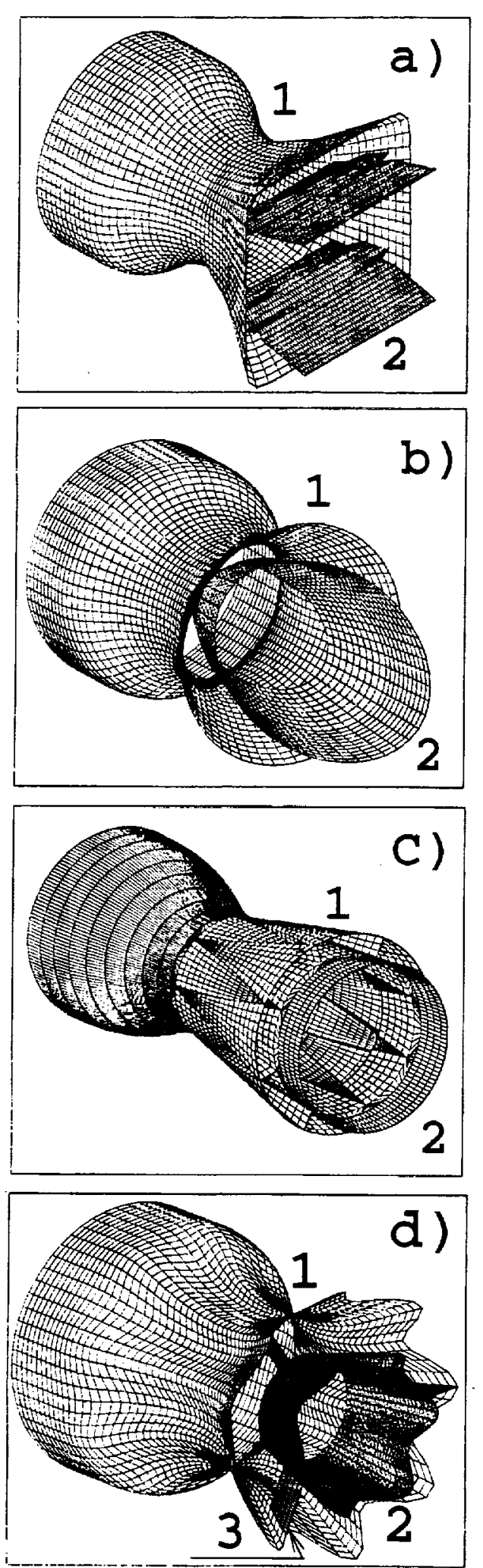

Figure 6 The Telescope nozzle embodiments: a- with the rectangular cross sections; b-with the elliptic cross section. c)- Chisel-Telescope nozzle; d)-Bluebell Telescope nozzle; The internal designs (number 2) are supported into the external designs (number 1 ) by the holders ( number 3).

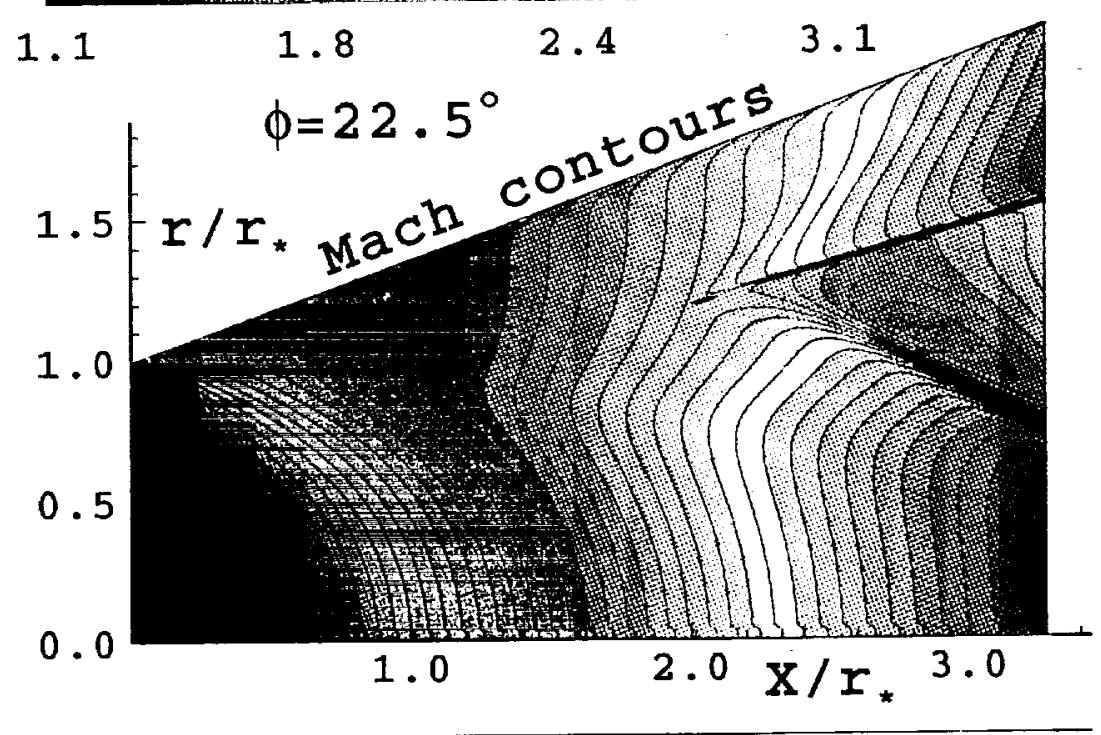

\section{Mach Contours}
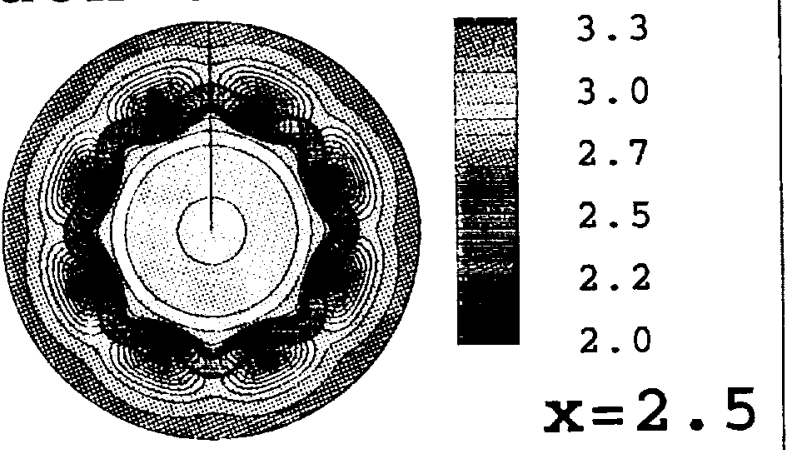

Mach Contours

c)

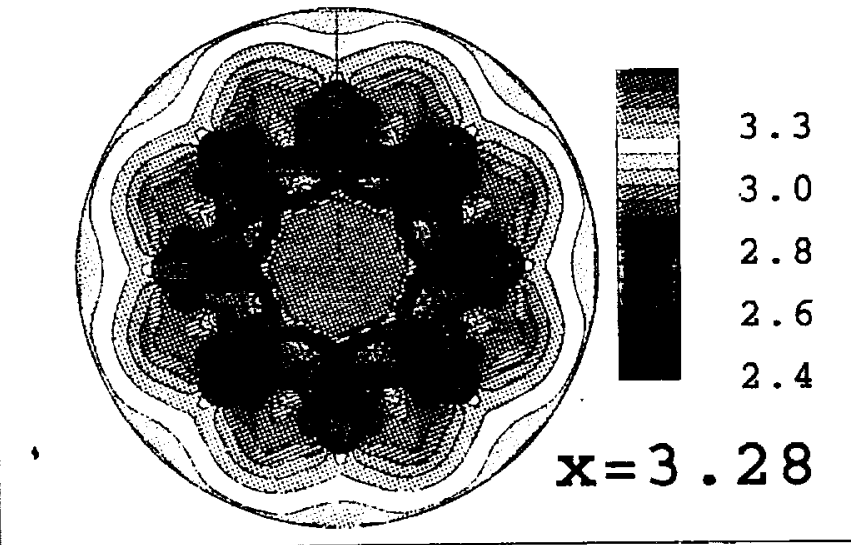

Figure 7. The Bluebell-Telescope nozzle with 8 corrugations of the internal design without petals. Mach contours: a)-inthe plane of symmetry $\mathrm{XOZ}$; b)-in cross section $x=2.5$, and $c$ )- in cross section at the nozzle exit, $\mathrm{x}=3.28$. 

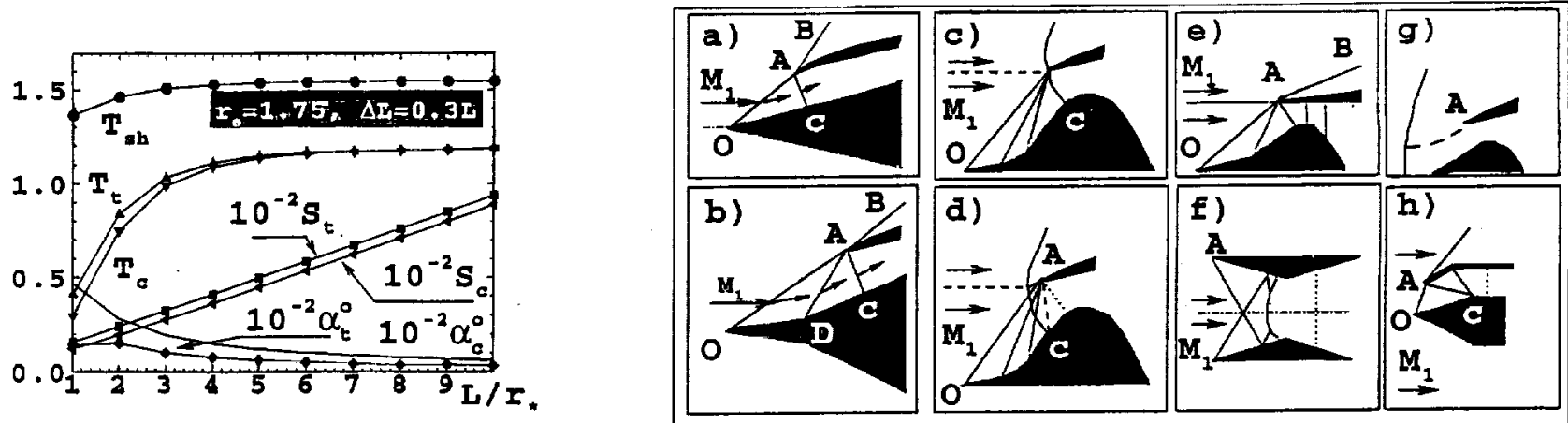

Figure 8 Conical Telescope nozzle: thrust, area, angles vs length divergent portion: $r_{e} 1.75, \Delta L=0.3 \mathrm{~L}$. Subscripts: "c"-for single conical nozzle; "sh"-total thrust; " $t$ "-for Telescope nozzle with one internal design. Symbols: T-thrust; $\alpha$-half-cone angle; S-lateral area.

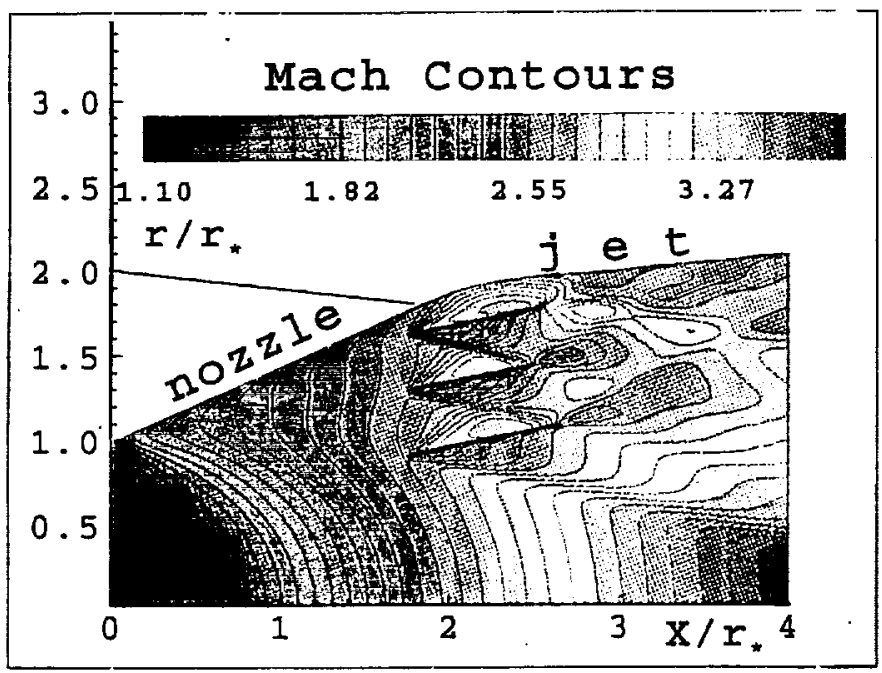

Figure 9. Conical Telescope nozzle with three internal plane designs at the nozzle exit. Mach contours.

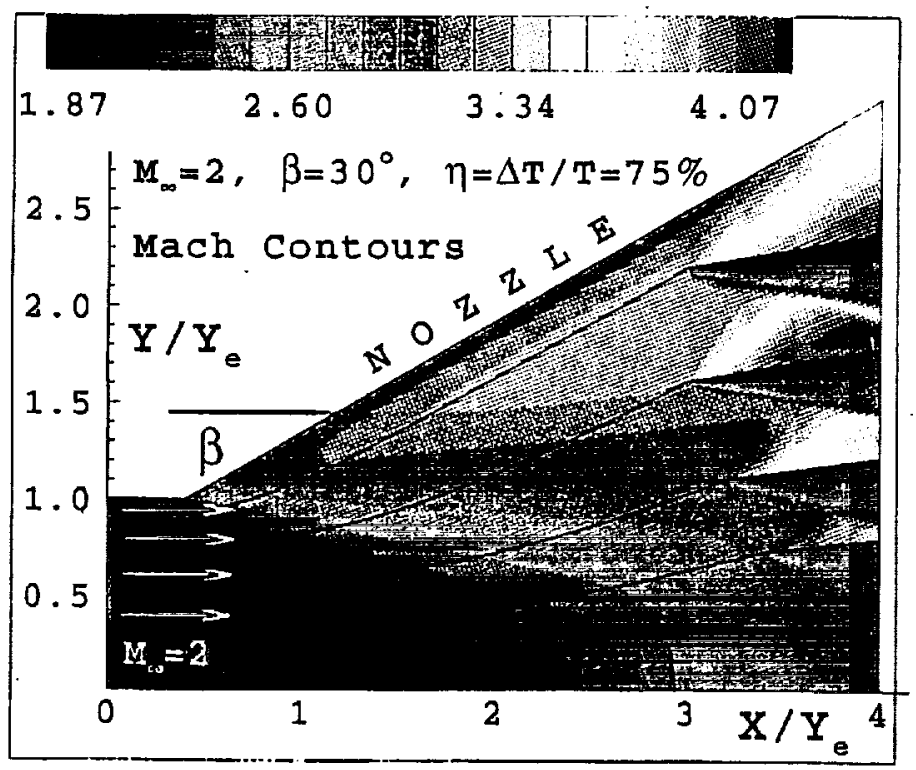

Figure 10. 2D Telescope nozzle with four internal designs at the exit. Mach contours.
Figure 11. Supersonic inlet schemes.

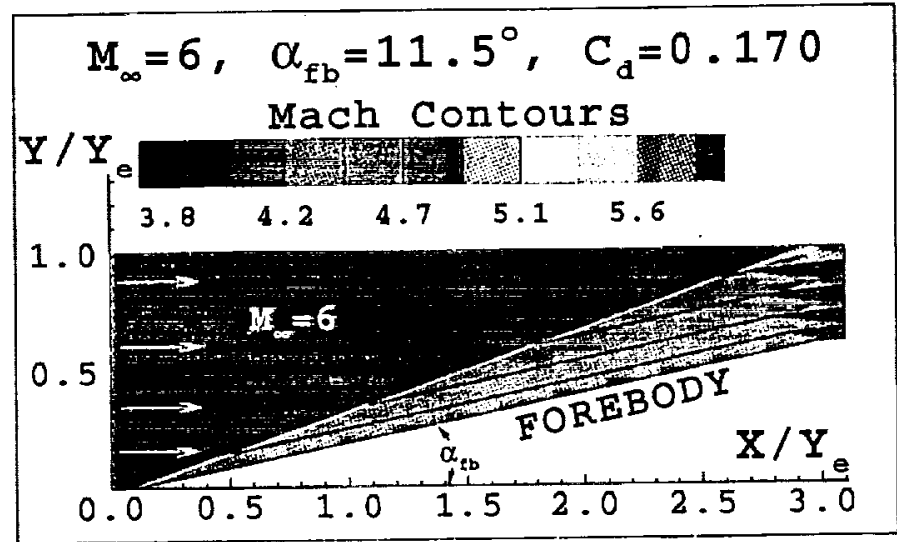

Figure 12. 2D Telescope inlet with the four internal designs.

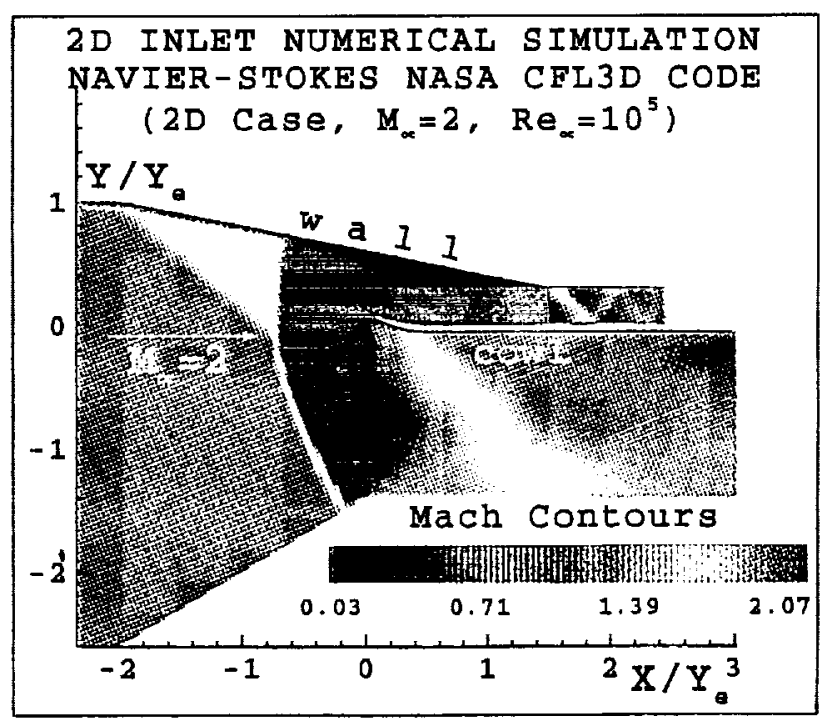

Figure 13 2D Telescope inlet with one internal design. Numerical simulation results based on full Navier-Stokes ecuations using NASA CFL3d code. 